\title{
Amenorrhea as a Side Effect of Low Dose Aripiprazole: An Adolescent Case
}

\author{
Ahmed Naguy \\ Al-Manara Child/Adolescent Psychiatry Centre, Kuwait Centre for Mental Health, Shuwaikh, Kuwait
}

\section{TO THE EDITOR}

Guler et al.' have recently reported an interesting case of amenorrhea in the setting of an adolescent girl with major depressive disorder (MDD) being treated with fluoxetine (FLUX) $40 \mathrm{mg}$ and aripiprazole (ARIP) $5 \mathrm{mg} /$ day. Few points are noteworthy mentioning here.

Although a modicum of evidence-base might support combination-initiation therapy in MDD, polypharmacy from the outset with an add-on AAP cannot be justified on clinical grounds especially in absence of psychosis or suicidality as the case portrays. Injudicious use of AAPs is currently rampant and sorely off-label driven. This practice is perilous and use is fraught with drastic cardio-metabolic and neuro-hormonal syndromes with child and adolescent psychiatric (CAP) population being at heightened risk by virtue of age. ${ }^{2)}$

ARIP is lauded for a metabolic-friendly profile, but this is not typically the case in CAP population.

True and rightful that ARIP at low dose has been deployed in clinical practice to treat risperidone-induced hyperprolactinemia (hPRL) through partial D2/D3 agonism and 5-HT2A antagonism releasing dopamine in the tubero-infundibular pathway. Whilst rare, yet reports of paradoxically ARIP-induced hPRL abound in literature. ${ }^{3)}$

Onset of hPRL can take place theoretically in 24 hours following a single dose of ARIP. Mechanistically, what is needed is to occupy $72 \%$ of $\mathrm{D} 2$ receptors in the tubero-infundibular pathway (anatomically projecting from arcu-

Received: September 12, 2018 / Accepted: November 14, 2018 Address for correspondence: Ahmed Naguy, MSc

Al-Manara Child/Adolescent Psychiatry Centre, Kuwait Centre for Mental Health, Jamal Abdul-Nassir St., Shuwaikh, Kuwait

Tel: +965-6554193, Fax: +965-24843900

E-mail: ahmednagy@hotmail.co.uk

ORCID: https://orcid.org/0000-0002-6465-456X ate nucleus to median eminence). ARIP is notorious to have D2 tenacity. This pharmacodynamics property can give rise to extrapyramidal syndromes and $\mathrm{hPRL}$ at these relatively small doses.

Selective serotonin reuptake inhibitors (SSRIs), by virtue of $5 \mathrm{HT} 2$ agonism can cause akathisia and hPRL on its own merits. ${ }^{4)}$ So, FLUX at $40 \mathrm{mg} /$ day in this case might have contributed to this side effect.

As indicated by authors, FLUX is a potent CYP 2D6 inhibitor and since ARIP is partly a substrate of 2D6 (and also 3A4). This 'combo' can push up ARIP levels causing higher blockade and accordingly likelihood of hPRL.

As there was no genotyping for the case, a remote possibility of 2D6 slow metabolizer (5-10\% of Caucasians) cannot be confidently ruled out/in and might contribute to the incident reported here.

Last but not least, high levels of anxiety as reflected in this case have been classically cited as a cause of hPRL in unmedicated subjects. ${ }^{5)}$

It behooves clinicians then to be more vigilant and cognizant of ARIP+SSRI combination in clinical practice. This 'combo' can be associated with higher D2 blockade and potential side effects.

\section{REFERENCES}

1. Guler G, Kutuk MO, Kara H. Amenorrhea as a side effect of low dose Aripiprazole: an adolescent case. Clin Psychopharmacol Neurosci 2018;16:343-345.

2. Naguy A, El-Sori D. Metformin for antipsychotic-related metabolic syndrome in children: fact or fiction? Chin Med J (Eng) 2018;131:1490-1491.

3. Naguy A. A comment on "Add-on Aripiprazole for atypical antipsychotic-induced, clinically significant hyperprolactinemia”. Indian J Psychol Med 2018;40:299-300.

4. Park YM. Serum prolactin levels in patients with major depressive disorder receiving selective serotonin reuptake inhibitor monotherapy for 3 months: a prospective study. Psychiatry

(ㄷ) This is an Open-Access article distributed under the terms of the Creative Commons Attribution Non-Commercial License (http://creativecommons.org/licenses/by-nc/4.0) which permits unrestricted non-commercial use, distribution, and reproduction in any medium, provided the original work is properly cited. 
144 A. Naguy

Investig 2017;14:368-371.

5. Sonino N, Navarrini C, Ruini C, Fallo F, Boscaro M, Fava GA.
Life events in the pathogenesis of hyperprolactinemia. Eur J Endocrinol 2004;151:61-65. 